\title{
Genome-wide association analyses for meat quality traits in Chinese Erhualian pigs and a Western Duroc $\times($ Landrace $\times$ Yorkshire) commercial population
}

Xianxian Liu', Xinwei Xiong ${ }^{\dagger}$, Jie Yang, Lisheng Zhou, Bin Yang, Huashui Ai, Huanban Ma, Xianhua Xie, Yixuan Huang, Shaoming Fang, Shijun Xiao, Jun Ren, Junwu Ma and Lusheng Huang*

\begin{abstract}
Background: Understanding the genetic mechanisms that underlie meat quality traits is essential to improve pork quality. To date, most quantitative trait loci (QTL) analyses have been performed on $F_{2}$ crosses between outbred pig strains and have led to the identification of numerous QTL. However, because linkage disequilibrium is high in such crosses, QTL mapping precision is unsatisfactory and only a few QTL have been found to segregate within outbred strains, which limits their use to improve animal performance. To detect QTL in outbred pig populations of Chinese and Western origins, we performed genome-wide association studies (GWAS) for meat quality traits in Chinese purebred Erhualian pigs and a Western Duroc $\times$ (Landrace $\times$ Yorkshire) $($ DLY) commercial population.

Methods: Three hundred and thirty six Chinese Erhualian and 610 DLY pigs were genotyped using the Illumina PorcineSNP60K Beadchip and evaluated for 20 meat quality traits. After quality control, 35985 and 56216 single nucleotide polymorphisms (SNPS) were available for the Chinese Erhualian and DLY datasets, respectively, and were used to perform two separate GWAS. We also performed a meta-analysis that combined P-values and effects of 29 516 SNPs that were common to Erhualian, DLY, $F_{2}$ and Sutai pig populations.

Results: We detected 28 and nine suggestive SNPs that surpassed the significance level for meat quality in Erhualian and DLY pigs, respectively. Among these SNPS, ss131261254 on pig chromosome 4 (SSC4) was the most significant $(P=7.97 \mathrm{E}-09)$ and was associated with drip loss in Erhualian pigs. Our results suggested that at least two QTL on SSC12 and on SSC15 may have pleiotropic effects on several related traits. All the QTL that were detected by GWAS were population-specific, including 12 novel regions. However, the meta-analysis revealed seven novel QTL for meat characteristics, which suggests the existence of common underlying variants that may differ in frequency across populations. These QTL regions contain several relevant candidate genes.

Conclusions: These findings provide valuable insights into the molecular basis of convergent evolution of meat quality traits in Chinese and Western breeds that show divergent phenotypes. They may contribute to genetic improvement of purebreds for crossbred performance.
\end{abstract}

\footnotetext{
* Correspondence: ma_junwu@hotmail.com; Lushenghuang@hotmail.com

${ }^{\dagger}$ Equal contributors

Key Laboratory for Animal Biotechnology of Jiangxi Province and the

Ministry of Agriculture of China, Jiangxi Agricultural University, Nanchang

330045, China
}

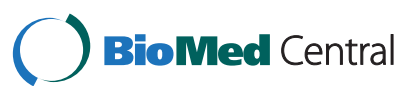

(c) 2015 Liu et al.; licensee BioMed Central. This is an Open Access article distributed under the terms of the Creative Commons Attribution License (http://creativecommons.org/licenses/by/4.0), which permits unrestricted use, distribution, and reproduction in any medium, provided the original work is properly credited. The Creative Commons Public Domain Dedication waiver (http://creativecommons.org/publicdomain/zero/1.0/) applies to the data made available in this article, unless otherwise stated. 


\section{Background}

Pork quality is an economically important trait in pig industry and has been one of the major objectives in pig breeding programs [1-3]. The main characteristics of meat include $\mathrm{pH}$, drip loss, colour, firmness, moisture content, marbling and intramuscular fat content $[4,5]$. Obviously, all meat quality traits are complex traits that depend on multiple interacting factors, including genetic background, which may depend on species, major genes and gene interaction, and environmental background, which may depend on feeding, management and slaughter conditions [6]. A few meat quality traits can be predicted from analyses on small muscle biopsies sampled from living animals. However, to meet the requirements for testing multiple meat quality traits, it is necessary to conduct post-mortem sampling from animal carcasses, which increases the overall cost of measurement. Some meat quality traits may have negative and antagonistic relationships with other traits such as growth rate. In addition, some meat quality traits show a low to moderate heritability [7], which reduces the effectiveness of traditional phenotype-based breeding strategies for which estimation of breeding values relies only on the phenotypes of relatives. However, with the availability of pig whole-genome sequence data and the rapid development of molecular genetics, much progress has been made in untangling the molecular mechanisms that underlie meat quality traits. For example, it has been shown that the genes insulin-like growth factor 2 (IGF2) [8,9], melano-cortin 4 receptor (MC4R) [9], protein kinase, AMP-activated, gamma 3 non-catalytic subunit (PRKAG3) [10] and ryanodine receptor 1 (RYR1) [11] have major effects on meat production and quality in pigs. Identification of these genes has greatly benefited the pig industry [12].

Since 1994 when Andersson et al. [6] first published the genetic mapping of quantitative trait loci (QTL) in pigs, numerous QTL have been reported and deposited in the Pig QTL database (http://www.animalgenome.org/cgibin/QTLdb/SS/index). However, most of these QTL were identified through family-based linkage analyses using a limited number of microsatellites and they span large genomic regions i.e. with a size greater than $15 \mathrm{cM}$, and considerable efforts are needed to refine the position of target QTL [13]. Recently, genome-wide association studies (GWAS) have emerged as a powerful new approach to identify QTL in livestock. By taking advantage of the highthroughput genotyping technologies (e.g. the Illumina Porcine60KSNP chip platform [14]) and of the much lower level of linkage disequilibrium (LD) in outbred populations than in resource families, GWAS has proven to be far more efficient to estimate location and effect of QTL than linkage analysis $[15,16]$. In addition, QTL linkage analyses are based on the assumption that alternative QTL alleles are fixed in each parental line of the experimental intercross. This is not the case for GWAS and the detected associations are based on the LD between SNPs and causative mutations [17].

To date, many porcine QTL have been mapped using crosses between outbred lines (e.g. Chinese breeds $x$ Western breeds), but there are few studies on QTL analyses in outbred populations. In this study, we conducted GWAS for meat quality traits in Chinese Erhualian and Western DLY [Duroc $\times($ Landrace $\times$ Yorkshire $)$ commercial populations. The Erhualian pig breed, like the Meishan breed, is a Chinese fat-type line that is well known for its high prolificacy, superior meat quality and strong resistance to harsh environments; thus, it is often included in Chinese pig breeding programs. Compared to the Erhualian breed, the DLY cross shows greater growth rate and meat productivity and currently has the biggest share of the pork market in China. Our aim was to map population-specific QTL at high resolution for meat quality traits in Chinese purebred Erhualian and western crossbred DLY populations.

Previously, we reported QTL scans using a White Duroc $\times$ Erhualian $F_{2}$ resource population and Chinese Sutai pigs and the identification of a number of QTL that affect meat quality traits [18-24]. The Sutai pig is a synthetic line that derives from a cross between the Duroc breed (50\%) and the Chinese Taihu (50\%), which itself includes Erhualian, Meishan and Fengjing pigs [25]. It has been shown that the joint analysis of multiple phenotypes can increase the power of QTL detection, thus we performed a meta-analysis of GWAS on four experimental populations i.e. Erhualian, DLY, $\mathrm{F}_{2}$ and Sutai.

\section{Methods}

\section{Ethics statement}

All procedures involving animals followed the guidelines for the care and use of experimental animals approved by the State Council of the People's Republic of China. The ethics committee of Jiangxi Agricultural University specifically approved this study.

\section{Animals and phenotypic traits}

This study involved two populations: Chinese Erhualian pigs and Western DLY pigs. The Erhualian population comprised 168 barrows and 168 gilts, which were born and raised for two to three months on Erhualian cooperatives that were located around the Jiaoxi Changzhou city in Jiangsu province. They were then transferred to a farm in Nanchang city in two batches. The DLY population consisted of 306 males and 304 females, which were raised on a farm in Xiushui city. All experimental animals were fed on a similar diet under a standardized feeding and management regimen, and given free access to water, and then slaughtered at approximately 300 days of age for Erhualian pigs and 180 days for DLY pigs in 
the same commercial abattoir. The meta-analysis that combined four GWAS included two additional populations: $\mathrm{F}_{2}$ and Sutai pigs, as described by Ma et al. [26]. Briefly, $1029 \mathrm{~F}_{2}$ pigs that originated from nine $\mathrm{F}_{1}$ boars and $59 F_{1}$ sows, which were the progeny of two White Duroc sires and 17 Erhualian dams, were slaughtered at $240 \pm 5$ days of age and were submitted to standardized cutting of the carcass. The Sutai population consisted of 461 offspring from four Sutai boars and 55 Sutai sows that were also slaughtered at $240 \pm 5$ days of age. The growth rates of these four populations differed in the following order: growth rate of DLY > F2 and Sutai > Erhualian pigs. They were all fed to similar market weight ( 90 kg) before slaughter and thus age at slaughter differed for each population.

Eight meat quality traits, including pH, EZ-drip loss, $L^{*}$ for lightness, $a^{*}$ for redness, $b^{*}$ for yellowness, moisture content, marbling and firmness were measured on the longissimus muscle (LM) between the 10th rib and the first lumbar vertebra and semimembranosus muscle (SM) from the left side of the carcass [1,19]. Meat characteristics of both LM and SM were measured for all Erhualian individuals, but for DLY individuals, only LM tissue samples were collected from carcasses due to the high cost of sampling. $\mathrm{pH}$ was measured twice on each sample using a Delta $320 \mathrm{pH}$ meter and the average value of the two measurements was used in subsequent analyses. For Erhualian individuals, $\mathrm{pH}$ values and $\mathrm{pH}$ drop were measured at $45 \mathrm{~min}$ and $24 \mathrm{~h}$ after slaughter in the morning. However, all DLY pigs were slaughtered between $11 \mathrm{pm}$ and $1 \mathrm{am}$. Based on measures from 30 DLY muscle samples, we observed that $\mathrm{pH}$ decreased within the first 24 hours post-mortem and was almost stabilized after 24 hours. Thus, to avoid night work, we measured $\mathrm{pH}$ for DLY individuals at $36 \mathrm{~h}$ post-mortem i.e. at $1 \mathrm{pm}$ on the third day. Drip loss was assayed using an EZ-Drip Loss method [27-29]. Meat colour scores (ranging from 1 to 6 , with $1=$ pale and $6=$ dark), marbling scores (ranging from 1 to 10 with $1=$ devoid and $10=$ overly abundant) and firmness scores (ranging from 1 to 5 ) were subjectively evaluated according to National Pork Producer Council (NPPC) guidelines [30]. Three colour parameters $\mathrm{L}^{*}$, $\mathrm{a}^{*}$ and $\mathrm{b}^{*}$ on the surface cuts of LM and SM were objectively evaluated with a CM-2600d/2500d Minolta Chroma meter. Moisture content was determined by the routine oven-drying method. Summary statistics for all traits investigated in the Erhualian and DLY populations are in Tables 1 and 2, respectively.

\section{Genotyping and quality control}

Genomic DNA was isolated from ear tissue using a standard phenol/chloroform method and dissolved in Tris-EDTA buffer. DNA quality and concentration were determined using a Nanodrop-1000 spectrophotometer (Thermo Fisher,
Table 1 Descriptive statistics of meat quality traits of longissimus muscle (LM) and semimembranosus muscle (SM) from Chinese Erhualian pigs

\begin{tabular}{|c|c|c|c|c|c|c|}
\hline Traits & $\mathbf{N}$ & Mean & $S D^{a}$ & Min. & Max. & $h^{2 c}$ \\
\hline \multicolumn{7}{|l|}{$\mathrm{pH}^{\mathrm{b}}$} \\
\hline LM_pH45min & 334 & 6.48 & 0.26 & 5.62 & 7.07 & 0.08 \\
\hline LM_pH24h & 332 & 5.99 & 0.42 & 5.37 & 7.03 & 0.41 \\
\hline LM_pHdrop_45min-24 h & 332 & 0.49 & 0.37 & -0.10 & 1.29 & 0.16 \\
\hline SM_pH45min & 334 & 6.42 & 0.28 & 5.66 & 7.13 & 0.07 \\
\hline SM_pH24h & 334 & 6.12 & 0.38 & 5.34 & 7.00 & 0.33 \\
\hline SM_pHdrop_45min-24 h & 334 & 0.31 & 0.35 & -0.10 & 1.42 & 0.08 \\
\hline \multicolumn{7}{|l|}{ Drip loss } \\
\hline LM_DripEZ_24h,\% & 333 & 1.14 & 1.14 & 0.18 & 6.13 & 0.12 \\
\hline SM_DripEZ_24h,\% & 331 & 0.87 & 0.80 & 0.18 & 6.49 & 0.05 \\
\hline \multicolumn{7}{|l|}{ Meat colour measures } \\
\hline LM_ColorM_L24h & 334 & 46.10 & 4.47 & 36.66 & 60.15 & 0.18 \\
\hline LM_ColorM_a24h & 333 & 2.61 & 1.43 & -0.28 & 7.38 & 0.12 \\
\hline LM_ColorM_b24h & 334 & 5.63 & 1.83 & 1.29 & 13.09 & 0.32 \\
\hline LM_ColorScore_24h (1-6) & 334 & 3.20 & 0.62 & 1.50 & 4.50 & 0.05 \\
\hline SM_ColorM_L24h & 334 & 39.39 & 3.52 & 31.14 & 51.25 & 0.25 \\
\hline SM_ColorM_a24h & 332 & 5.29 & 1.49 & 1.91 & 9.58 & 0.31 \\
\hline SM_ColorM_b24h & 332 & 4.86 & 2.08 & 0.75 & 11.47 & 0.56 \\
\hline SM_ColorScore_24h (1-6) & 334 & 4.09 & 0.54 & 2.0 & 5.5 & 0.35 \\
\hline \multicolumn{7}{|l|}{ Moisture content } \\
\hline LM_MoistureContent, \% & 332 & 73.63 & 1.79 & 66.32 & 79.71 & 0.18 \\
\hline \multicolumn{7}{|l|}{ Subjective scores } \\
\hline LM_Firmness (1-5) & 334 & 2.17 & 0.51 & 1.0 & 3.5 & 0.36 \\
\hline LM_Marbling (1-10) & 331 & 3.95 & 1.90 & 1.5 & 10.0 & 0.29 \\
\hline SM_Marbling (1-10) & 334 & 2.16 & 0.58 & 1.5 & 5.0 & 0.05 \\
\hline
\end{tabular}

${ }^{\mathrm{a} S t a n d a r d ~ d e v i a t i o n ;}{ }^{\mathrm{b}} \mathrm{pH}$ was measured on samples of LM and SM 45 min and $24 \mathrm{~h}$ post-mortem; ' heritability estimates.

USA). A total of 336 Chinese Erhualian and 610 DLY pigs were genotyped for 61565 SNPs using the Illumina PorcineSNP60K Beadchip according to the manufacturers protocol. Quality control procedures were carried out to remove SNPs with a call rate greater than $90 \%$, a minor allele frequency (MAF) less than 0.01 and a significant deviation from Hardy-Weinberg equilibrium $\left(P \leq 10^{-5}\right)$; moreover, animals with more than $10 \%$ missing genotypes or more than $5 \%$ Mendelian errors were removed from the dataset. A final set of 35985 SNPs for 331 Chinese Erhualian and 56216 SNPs for 610 DLY pigs were used for further statistical analysis. The large difference in number of SNPs that were retained for each population is explained by the fact SNPs on the Illumina PorcineSNP60K Beadchip were primarily ascertained based on a small panel of western pig genomes and that more SNPs had a MAF less than 0.01 for the Chinese Erhualian dataset than for western DLY dataset. SNP chromosomal positions 
Table 2 Descriptive statistics of meat quality traits of longissimus muscle (LM) from Western DLY pigs ${ }^{1}$

\begin{tabular}{lcccccc}
\hline Traits & N & Mean & S.D. & Min. & Max. & $\mathbf{h}^{\mathbf{2}}$ \\
\hline pH & & & & & & \\
LM_pH36h & 580 & 5.50 & 0.25 & 5.04 & 6.42 & 0.34 \\
$\begin{array}{l}\text { Drip loss } \\
\text { LM_DripEZ_36h, \% }\end{array}$ & 583 & 3.35 & 2.09 & 0.15 & 9.52 & 0.42 \\
Meat colour measures & & & & & & \\
LM_ColorM_L36h & 584 & 47.03 & 3.31 & 34.58 & 58.52 & 0.37 \\
LM_ColorM_a36h & 584 & 1.51 & 1.11 & -1.45 & 6.62 & 0.40 \\
LM_ColorM_b36h & 584 & 5.68 & 1.47 & 1.38 & 9.21 & 0.34 \\
LM_ColorScore_36h(1-6) & 584 & 3.05 & 0.81 & 1.0 & 4.5 & 0.35 \\
Moisture content & & & & & & \\
LM_MoistureContent, \% & 610 & 75.00 & 0.71 & 72.00 & 78.00 & 0.27 \\
Subjective scores & & & & & & \\
LM_Firmness (1-5) & 575 & 2.86 & 0.73 & 1.0 & 5.0 & 0.19 \\
LM_Marbling (1-10) & 610 & 2.73 & 0.64 & 1.5 & 5.5 & 0.18 \\
\hline
\end{tabular}

${ }^{1}$ See footnotes in Table 1.

were based on the current pig genome assembly (Sus Scrofa Build 10.2 assembly).

\section{Statistical analyses}

Heritability of meat quality traits was estimated using the polygenic function of GenABEL v1.7 [31]. Genomewide association studies (GWAS) analyses were performed using polygenic and mmscore function of GenABEL v1.7 [31]. Associations between SNPs and phenotypic values were evaluated using a generalized linear mixed model [32,33], which accounted for population structure by fitting the covariance among individuals inferred from high-density SNP data. The variance-covariance matrix was proportional to genome-wide identity-by-state [34]. Sex and batch were fitted as fixed effects in the model. Bonferroni-corrected thresholds of $0.05 / \mathrm{N}$ and $1 / \mathrm{N}$, where $\mathrm{N}$ is the number of SNPs used for the analyses, were adopted for the 5\% genome-wide and suggestive significance, respectively [35,36]. After quality control, the genome-wide and suggestive significance thresholds were 1.38E-06 (0.05/35 985) and 2.77E-05 (1/35 985), respectively for the Chinese Erhualian dataset, and $8.89 \mathrm{E}-07$ $(0.05 / 56216)$ and $1.78 \mathrm{E}-05$ (1/56216), respectively for the DLY dataset. In addition, a less stringent threshold of 1.00E-04 was applied in order to detect moderate associations and possible pleiotropic effects of QTL on correlated traits.

Since population stratification is generally considered as a major factor that influences GWAS results [37], it was assessed by examining the distribution of test statistics generated from the thousands of association tests and assessing their deviation from the null distribution (i.e. the distribution expected under the null hypothesis of no SNP associated with the trait) in quantile-quantile (Q-Q) plots [37]. The Q-Q plots were generated using $\mathrm{R}$ software.

A meta-analysis was performed using a Z-score approach in the METAL software [38] that combines $P$-values and effects of a set of 29516 SNPs that were common to all four populations i.e. Erhualian, DLY, $F_{2}$ and Sutai.

\section{Results}

\section{Assessment of population stratification}

In this study, we conducted two separate single-population GWAS i.e. on Erhualian and DLY populations and a metaanalysis of GWAS on four populations. The Q-Q plots of the test statistics for each separate GWAS and the metaanalysis are in Figure S1 [See Additional file 1: Figure S1] and Figure S2 [See Additional file 2: Figure S2], respectively. Average genomic inflation factors $(\lambda)$ for each GWAS were equal to 1.01 and 1.04 for the Erhualian and DLY populations, respectively, and to 1.08 for the combined populations, which indicated the absence of any obvious population stratification.

\section{Results of the separate GWAS for the Erhualian and DLY populations}

Overall, we identified 37 SNPs (one unmapped SNP) on 14 chromosomes that were significantly associated with $\mathrm{pH}$, drip loss, meat colour, moisture content and firmness in these populations (Table 3), which demonstrates the diverse genetic architecture of meat quality traits in pigs. Among these 37 associations, 28 were identified for the Erhualian population and nine for the DLY population; only one SNP (ss131261254 on SSC4 for LM_DripEZ_24h trait in the Erhualian dataset) exceeded the 5\% genomewide significance level $(P=7.97 \mathrm{E}-09)$. No SNP was associated with marbling at the GWAS significance level. Manhattan plots of GWAS for the 20 meat quality traits that were analyzed in the two populations are in Figure 1 and Figure S3 [See Additional file 3: Figure S3].

\section{$\mathrm{pH}$ values and drip loss}

We identified three significant SNPs associated with $\mathrm{pH}$ values in the Erhualian dataset, including one for LM_pH24h, one for SM_pH24h and one for LM pHdrop_45min-24 h, which were localized on SSC9 (SSC for Sus scrofa chromosome), SSC3 and SSC16, respectively. No significant SNP was associated with $\mathrm{pH}$ value in the DLY dataset. Five significant SNPs were associated with drip loss in the Erhualian dataset, including four SNPs for drip loss of LM on SSC4 and one SNP for drip loss of SM on SSC1. In the DLY dataset, two SNPs associated with drip loss of LM were identified on SSC13 and SSC9, respectively. No common QTL was found between the two populations. 
Table 3 Description of SNPs significantly associated with meat quality traits in Erhualian and DLY pigs

\begin{tabular}{|c|c|c|c|c|c|c|c|c|c|}
\hline Traits $^{2}$ & Peak SNP & No. $^{3}$ & Chr & Pos $(b p)^{4}$ & $\mathrm{PPL}^{5}$ & Nearest genes ${ }^{6}$ & Freq $^{7}$ & Effects $^{8}$ & $P$-value ${ }^{9}$ \\
\hline \multicolumn{10}{|l|}{$\overline{\mathrm{EHL}^{1}}$} \\
\hline LM_pH24h & ss131409440 & 1 & 9 & 14439917 & & INTS7 & 0.67 & 0.088 & $9.78 \mathrm{E}-06$ \\
\hline SM_pH24h & ss131225834 & 1 & 3 & 1527192 & & WBSCR17 & 0.33 & 0.185 & $3.13 \mathrm{E}-06$ \\
\hline LM_pHdrop_45min-24 h & ss107857062 & 1 & 16 & 7949030 & c & SEMA5A & 0.53 & 0.158 & $8.05 \mathrm{E}-06$ \\
\hline LM_DripEZ_24h & ss131261254 & 1 & 4 & 4433660 & & ENSSSCG00000019169 & 0.15 & 0.686 & 7.97E-09 \\
\hline LM_DripEZ_24h & ss478940762 & 2 & 4 & 35761053 & & RIMS2 & 0.12 & 0.618 & $2.62 \mathrm{E}-06$ \\
\hline LM_DripEZ_24h & ss131270350 & 1 & 4 & 85151430 & & ST18 & 0.14 & 1.092 & $6.26 \mathrm{E}-06$ \\
\hline SM_DripEZ_24h & ss131134976 & 1 & 1 & 181797733 & & MEGF11 & 0.50 & 0.247 & $2.31 \mathrm{E}-06$ \\
\hline LM_ColorM_a24h & ss131341943 & 1 & 7 & 31500144 & & KLHL31 & 0.60 & 0.505 & $9.1 \mathrm{E}-06$ \\
\hline LM_ColorM_a24h & ss478937995 & 3 & 12 & 58130567 & b & MYH3 & 0.18 & 0.945 & $1.04 \mathrm{E}-05$ \\
\hline LM_ColorM_a24h & ss131565824 & 1 & 5 & 2588817 & & EFCAB6 & 0.65 & 0.545 & $1.18 \mathrm{E}-05$ \\
\hline LM_ColorM_b24h & ss120027962 & 1 & 14 & 11463494 & & BNIP3L & 0.02 & 1.628 & $3.64 \mathrm{E}-06$ \\
\hline LM_ColorM_b24h & ss478937995 & 2 & 12 & 58130567 & $b$ & MYH3 & 0.18 & 0.976 & $1.65 \mathrm{E}-05$ \\
\hline LM_ColorScore_24h & ss131352394 & 1 & 7 & 5406955 & a & EEFIE1 & 0.74 & 0.234 & 5.49E-06 \\
\hline LM_ColorScore_24h & ss131461879 & 1 & 12 & 36158023 & & SUPT4H1 & 0.63 & 0.208 & $2.29 \mathrm{E}-05$ \\
\hline SM_ColorM_b24h & ss107878019 & 1 & 12 & 9939154 & & ENSSSCG00000025361 & 0.95 & 1.435 & $1.88 \mathrm{E}-05$ \\
\hline SM_ColorScore_24h & ss478943784 & 1 & 17 & 56358556 & & SULF2 & 0.33 & 0.235 & 7.70E-06 \\
\hline LM_MoistureContent & ss131186989 & 1 & 2 & 43911957 & & $\angle D H A$ & 0.01 & 1.010 & $5.18 \mathrm{E}-06$ \\
\hline LM_MoistureContent & ss131458048 & 1 & 12 & 24427829 & & NFE2L1 & 0.01 & 0.860 & 5.31E-06 \\
\hline LM_MoistureContent & ss131054125 & 1 & 8 & 14014229 & & ENSSSCG00000029791 & 0.51 & 0.653 & 8.07E-06 \\
\hline LM_MoistureContent & ss131538960 & 2 & 16 & 74982852 & c & MFAP3 & 0.86 & 1.112 & 1.67E-05 \\
\hline LM_Firmness & ss131096544 & 2 & 12 & 57123997 & b & WDR16 & 0.72 & 0.232 & 4.10E-06 \\
\hline LM_Firmness & ss478937524 & 1 & 7 & 7764396 & a & TMEM14C & 0.64 & 0.225 & $1.81 \mathrm{E}-05$ \\
\hline \multicolumn{10}{|l|}{$D_{L} Y^{1}$} \\
\hline LM_DripEZ_36h & ss131494523 & 1 & 13 & 141530003 & & FAM43A & 0.36 & 0.435 & $1.53 \mathrm{E}-05$ \\
\hline LM_DripEZ_36h & ss131400253 & 1 & 9 & 95957104 & $d$ & SNX13 & 0.88 & 0.740 & 1.57E-05 \\
\hline LM_ColorM_a36h & ss107833468 & 1 & 15 & 134397712 & e & RESP18 & 0.37 & 0.245 & $1.78 \mathrm{E}-05$ \\
\hline LM_ColorM_b36h & ss107856037 & 1 & 15 & 92671886 & & AGPS & 0.32 & 0.569 & 9.17E-06 \\
\hline LM_ColorScore_36h & ss131398982 & 1 & 9 & 84832035 & $d$ & ASNS & 0.62 & 0.122 & $4.78 \mathrm{E}-06$ \\
\hline LM_ColorScore_36h & ss131528409 & 1 & 15 & 127419860 & e & IKZF2 & 0.56 & 0.153 & $7.27 \mathrm{E}-06$ \\
\hline LM_ColorScore_36h & ss107897208 & 1 & 9 & 45577347 & & NCAM1 & 0.44 & 0.148 & 9.96E-06 \\
\hline LM_ColorScore_36h & ss107799219 & 1 & 0 & 0 & & & 0.98 & 0.543 & $1.46 \mathrm{E}-05$ \\
\hline LM_Firmness & ss131184920 & 1 & 2 & 34977540 & & KIF18A & 0.37 & 0.172 & $1.47 \mathrm{E}-05$ \\
\hline
\end{tabular}

${ }^{1}$ Chinese Erhualian indigenous population and Western Duroc $\times$ (Landrace $\times$ Yorkshire) commercial population; ${ }^{2}$ description of the traits is in Table $1 ;{ }^{3}$ number of SNPs that surpassed the suggestive significance level within the QTL regions; ${ }^{4}$ positions of the most significant SNP according to the Sus Scrofa Build 10.2 assembly; ${ }^{5}$ possible pleiotropic loci (PPL) were indicated by common letters on different rows; ${ }^{6}$ annotated genes nearest to the most significant SNP; gene names starting with ENSSSCG follow the Ensembl nomenclature while other gene symbols follow the HUGO nomenclature; 7,8 frequencies and effects of the allele that increases phenotype value in the two populations; ${ }^{9}$ genome-wide significant associations are underlined.

\section{Meat colour}

Twelve and six significant SNPs associated with meat colour parameters were identified in the Erhualian and DLY datasets, respectively. In the Erhualian dataset, the most significant SNP was ss131341943 on SSC7 for LM_ColorM_a24h; SNP ss478937995 at 58.13 Mb on SSC12 was associated with both LM_ColorM_a24h and LM_ColorM_b24h. In addition, SNP ss131461879 at $36.15 \mathrm{Mb}$ on SSC12 and SNP ss131352394 on SSC7 were shown to have effects on LM_ColorScore_24h in this dataset. SNPs for SM_ColorM_b24h and SM ColorScore_24h were identified on SSC12 and SSC17, respectively. We did not detect any common region for the same trait on LM and SM. However, except for one unmapped SNP, all SNPs that were associated with meat colour of LM in the DLY dataset were located on SSC9 and SSC15. SNP ss107833468 for LM_ColorM_a36h at 134.39 $\mathrm{Mb}$ on SSC15 is the most significant SNP and is 


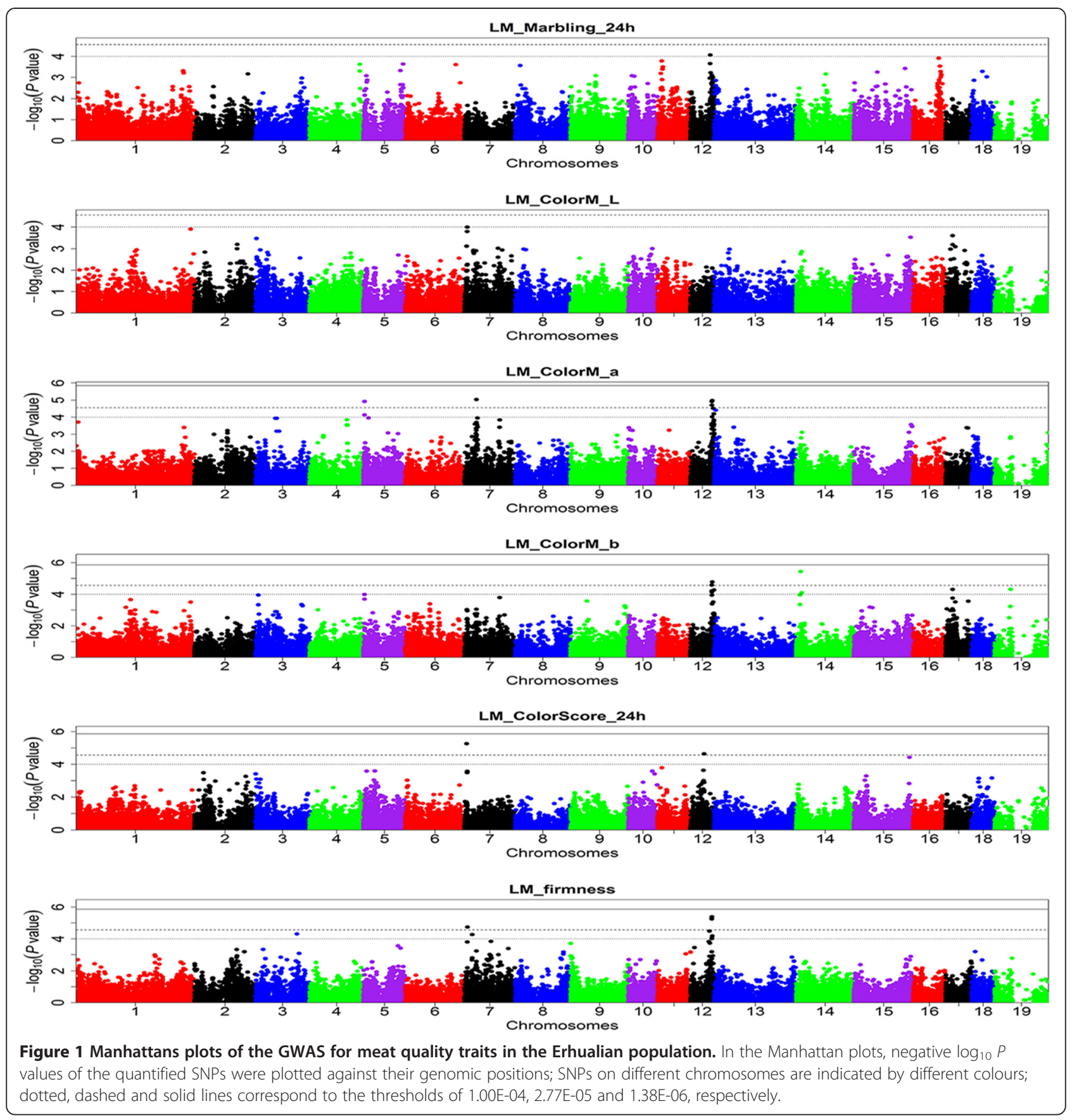

located near SNP ss131528409 at $127.41 \mathrm{Mb}$ for LM ColorScore_36h. The other two SNPs for LM_ColorScore 36h were detected on SSC9.

Moisture content and firmness scores

In the Erhualian dataset, five and three SNPs were significantly associated with LM_MoistureContent and LM_Firmness, respectively. All the SNPs associated with LM_MoistureContent are located in four QTL regions on SSC2, SSC8, SSC12 and SSC16. In the DLY dataset, no significant SNP was associated with LM_MoistureContent and only one SNP ss131184920 on SSC2 was detected for LM_Firmness.

Loci detected by the meta-analysis of GWAS across four populations

Meta-analysis is a powerful method that can reveal otherwise hidden or unclear associations that are detected by independent studies [38]. Our meta-analysis of GWAS across four populations revealed 16 SNPs associated with different meat quality traits: four for LM_pH24h, one for LM_DripZ_24h, one for LM_ColorScore_24h, five for 
LM_MoistureContent, one for LM_ColorM_a24h, two for LM_ColorM_b24h and two for LM_ColorM_L24h (Table 4 and Figure S4 [See Additional file 4: Figure S4]). Among these 16 SNPs, the most significant association was observed for SNP ss131289803 on SSC5 for LM_MoistureContent $(P=2.52 \mathrm{E}-07)$.

\section{Discussion}

Comparison between the QTL identified in the current and previous studies

A large number of QTL have been identified in multiple $\mathrm{F}_{2}$ intercrosses between Chinese and Western outbred pig strains, but few QTL have been detected within the founder strains (particularly the Chinese indigenous pig breeds), which restricts the use of maker-assisted selection to improve the performance of the founder animals and that of the crossbred animals. Here, we analyzed replicability of GWAS results between intercrosses and Chinese- and Western-type outbred populations. In the Erhualian dataset, we found a significant SNP ss131225834 for SM_pH24h at 15.27 Mb on SSC3 (Table 3), which is close to a locus that was previously detected for the same trait in Sutai pigs [26]. In addition, three significant SNPs for colour $\mathrm{a}^{*}$ of LM were identified in a region around $58.13 \mathrm{Mb}$ on SSC12, which confirms the locus reported by Luo et al. [39] for a Large White $\times$ Minzhu $F_{2}$ intercross population. Thus, our results highlight several QTL that segregate in both $F_{2}$ intercrosses and Chinese purebred Erhualian pigs. The position of these QTL could be further refined since the extent of LD is smaller in Chinese breeds than in western breeds and intercrosses $[40,41]$.

\section{Detection of novel QTL}

Our GWAS results not only confirmed a number of previously reported QTL but also revealed 12 novel loci. Six of these 12 loci were detected using Erhualian pig data, including: ss131409440 on SSC9 for LM_pH24h, ss478943784 on SSC17 for SM_ColorScore_24h, ss131186989 on SSC2, ss131054125 on SSC8 and ss131538960 on SSC16 for LM_MoistureContent, ss131096544 on SSC12 for LM_Firmness. The remaining six loci were identified using DLY pig data, including ss131494523 on SSC13 and ss131400253 on SSC9 for LM_DripEZ_36h, ss107856037 on SSC15 for LM_ColorM_b36h, ss131398982 and ss107897208 on SSC9 for LM_ColorScore_36h and ss131184920 on SSC2 for LM_Firmness.

\section{Possible pleiotropic QTL}

Our results indicate that some regions may have pleiotropic effects on different meat quality traits. Using the Erhualian pig data, a $2.36 \mathrm{Mb}$ region (between 5.40 and $7.76 \mathrm{Mb}$ ) on SSC7 was shown to contain SNPs that are associated with three related traits, including the most significant SNP ss120019062 for LM_ColorM_L24h at $7.02 \mathrm{Mb} \quad(P=1.01 \mathrm{E}-04)$, SNP ss131352394 for LM_ ColorScore_24h at $5.41 \mathrm{Mb}$ and SNP ss478937524 for LM_Firmness at $7.76 \mathrm{Mb}$ (Figure 1 and Table 3). On SSC12, SNP ss478937995 at $58.13 \mathrm{Mb}$ was associated both with LM_ColorM_a24h and LM_ColorM_b24h and is close to SNP ss131096544 at $57.12 \mathrm{Mb}$ that is associated with LM_Firmness. Interestingly, the most significant SNP, ss131466676 for LM_Marbling_24h $(P=8.59 \mathrm{E}-05)$, was also identified at $52.72 \mathrm{Mb}$ on SSC12 (Figure 1), although it did not reach the suggestive significance level. This relatively lower significance might be due to the lack of precision in phenotypic measurement, the frequency and the size of the effect of the allele, and the number of loci that affect the trait. Coincidently, Luo et al. [39] also reported several significant SNPs for intramuscular fat content (IMF), marbling and meat colour on SSC12 in the proximal region between 46.90 and $55.22 \mathrm{Mb}$. In addition, the most significant SNPs for $\mathrm{pH}$ drop and moisture

Table 4 Description of SNPs significantly associated with meat quality traits in the meta-analysis of four pig populations (Erhualian, DLY, $F_{2}$ and Sutai) ${ }^{1}$

\begin{tabular}{|c|c|c|c|c|c|c|}
\hline Traits & Peak SNP & No. & Chr & Pos (bp) & Nearest genes & $P$-value \\
\hline LM_pH24h & ss131233621 & 1 & 3 & 136625292 & ID2 & $3.63 \mathrm{E}-06$ \\
\hline LM_pH24h & ss107835446 & 2 & 7 & 115457433 & ENSSSCG00000018967 & $9.39 \mathrm{E}-06$ \\
\hline LM_pH24h & ss131101693 & 1 & $x$ & 141793169 & HAUS7 & 2.46E-05 \\
\hline LM_DripEZ_24h & ss131380751 & 1 & 8 & 143349693 & WDFY3 & 1.20E-05 \\
\hline LM_ColorM_L24h & ss131499292 & 2 & 14 & 118515539 & PI4K2A & $1.91 \mathrm{E}-05$ \\
\hline LM_ColorM_a24h & ss131529154 & 1 & 15 & 133970166 & CDK5R2 & 5.62E-06 \\
\hline LM_ColorM_b24h & ss131233621 & 1 & 3 & 136625292 & ID2 & 1.04E-05 \\
\hline LM_ColorM_b24h & ss107823551 & 1 & 7 & 94809941 & ENSSSCG00000002270 & $2.12 \mathrm{E}-05$ \\
\hline LM_ColorScore_24h & ss131508309 & 1 & 14 & 18052581 & ENSSSCG00000021805 & 1.16E-05 \\
\hline LM_MoistureContent & ss131289803 & 1 & 5 & 68572410 & PARP11 & $2.52 \mathrm{E}-07$ \\
\hline LM_MoistureContent & ss107806758 & 4 & 7 & 35177641 & SPDEF & 5.87E-06 \\
\hline
\end{tabular}

${ }^{1}$ See footnotes in Table 3. 
content of LM in the Erhualian dataset were located within a region of approximately $4.51 \mathrm{Mb}$ (between74.98 and 79.49 Mb) on SSC16 (Table 3 and Figure S3).

For the DLY pig data, a $6.98 \mathrm{Mb}$ region (between 127.41 and $134.39 \mathrm{Mb}$ ) on SSC15 was associated with LM_ColorM_a36h and LM_ColorScore_36h. The correlation coefficient between drip loss and colour score of LM was negative and highly significant $\left(\mathrm{r}=-0.62, P<10^{-4}\right)$; two adjacent SNPs associated with both traits were within a region of $11.12 \mathrm{Mb}$ (between 84.83 and $95.95 \mathrm{Mb}$ ) on SSC9, which indicates that this region may contain a pleiotropic QTL (Table 3). Since the Bonferroni-corrected thresholds applied in our study are very stringent, it is likely that some QTL are missed if their effects are not strong enough. For example, if this threshold is reduced to $10^{-4}$, a $1.98 \mathrm{Mb}$ region (between 36.07 and $38.05 \mathrm{Mb}$ ) on SSC7 is detected that contains several SNPs associated with LM_DripEZ_36h $(P=1.01 \mathrm{E}-04)$, LM_ColorM_L36h $(P=2.25 \mathrm{E}-05)$ and LM_ColorScore_36h $(P=3.44 \mathrm{E}-05)$ for the DLY pig data [See Additional file 3: Figure S3].

\section{All loci identified by the GWAS are population-specific or tissue-specific}

The results that we obtained for the separate GWAS on the Erhualian and DLY populations did not reveal any common loci between these two pig breeds (Table 3), which suggests that major genes that lead to the marked phenotypic variations within the Erhualian and the DLY populations are distinct. However, it cannot be excluded that the two populations share a few of the minor genes that affect these traits. Moreover, our results showed clear differences in the genetic loci that influence meat quality traits between LM and SM in Erhualian pigs, which suggest that these two muscle tissues have distinct metabolic characteristics and contractile properties.

\section{Impact of the difference in phenotype ascertainment across populations on the results of the meta-analysis}

It has been suggested that a difference in the approaches used to dissect phenotypes across populations can reduce the power of statistical tests in a meta-analysis [42]. In our study, there was a certain phenotypic heterogeneity. First, DLY, $F_{2}$, Sutai and Erhualian pigs were slaughtered at different ages, i.e. 180, 240, 240 and 300 days, respectively, which correspond to the different time lengths required for the animals to grow to market weight. We consider that it is appropriate to measure meat quality in pigs at market weight and that the phenotypic data from these populations is comparable and can be used for meta-analysis. For the DLY pigs, due to practical difficulties, we conducted meat quality measurements at $36 \mathrm{~h}$ post-mortem while they were performed at $24 \mathrm{~h}$ post-mortem for the other pig populations. However, we found that phenotypic values at $24 \mathrm{~h}$ and $36 \mathrm{~h}$ post-mortem were approximately the same and thus, the level of phenotypic heterogeneity observed in this study is probably low.

Furthermore, unlike the direct analysis of pooled individual-level data, a meta-analysis uses the summary statistics from individual studies as the data points. Here, we applied a Z-score approach that combined P-values and the direction of effects observed in each population. This method can greatly alleviate the interference of different design factors from individual studies. Therefore, there may be an effect due to the different protocols used to dissect phenotypes among the test populations on the results of meta-analysis, but it is probably limited.

\section{Comparison of the loci identified by the meta-analysis with those by the single-population GWAS}

The meta-analysis involved four populations: Erhualian, DLY hybrid, $F_{2}$ (White Duroc $\times$ Erhualian) and Sutai (Duroc $\times$ Taihu) pigs. Both $F_{2}$ and Sutai pigs share some genetic background with Duroc and Erhualian pigs, thus these populations may harbour some common QTL alleles. Moreover, meta-analysis is a tool for aggregating information from multiple independent studies so that it can provide more power to identify associated variants with small effect sizes or low frequencies. Therefore, this meta-analysis enabled us to identify several novel loci that were not detected by separate single-population GWAS. In addition to the SNP on SSCX that was detected using the $F_{2}$ pig data, three additional SNPs for LM_pH24h on SSC3 and SSC7 were only detected by the meta-analysis. SNP ss131380751 on SSC8 for LM_DripEZ, SNP ss131499292 on SSC14 for LM_ColorScore_24h, SNP ss131289803 on SSC5 for LM_MoistureContent and SNP ss131233621 on SSC3 for LM_ColorM b24h were also only detected by the meta-analysis. The most significant SNP ss131233621 on SSC3 was the first SNP found to be associated with both LM_ColorM_b24h and LM_pH24h. Compared to independent analyses, the joint analysis enabled us to obtain more precise locations and stronger significance for some QTL. For example, the most significant SNP ss131529154 for LM_ColorM_a24h that was revealed by the meta-analysis (Table 4), was located at $133.97 \mathrm{Mb}$ on SSC15, which was closer to the known causative gene PRKAG3 at $133.80 \mathrm{Mb}$, than the most significant SNP ss107833468 (at $134.39 \mathrm{Mb}$ ) identified by the GWAS on the DLY population only. The $P$-value for the locus for LM_ColorM_a24h on SSC15 was smaller $(P=5.62 \mathrm{E}-06)$ in the combined analysis than in the analysis with the DLY $(P=1.78 \mathrm{E}-05)$ population only (Table 3). None of the single-population GWAS detected significant SNPs for LM_ColorM_L24h; however, two significant SNPs on SSC14 were detected by the meta-analysis ([See Additional file 4: Figure S4] and Table 4). These results demonstrate that meta-analysis of 
GWAS has additional power to identify common variants across populations.

It should be noted that, in general, allelic effect direction of the above-mentioned SNPs that showed a higher significance in the meta-analysis was consistent across all tested populations. In contrast, the level of significance of SNP ss478940762 for drip loss on SSC4 decreased in the meta-analysis (5.89E-05) compared to the GWAS using only the Erhualian population (2.62E-06), which may be caused by opposite allelic directions of this SNP in different populations.

\section{Plausible candidate genes at the identified loci}

We searched for promising candidate genes with functional relevance to the studied traits in an interval of $1 \mathrm{Mb}$ centred on the most significant SNP at each significant locus. Based on the Erhualian dataset, the PPP2R5A gene located at about $270 \mathrm{~kb}$ upstream of the most significant SNP (ss131409440) is the best candidate gene for LM_pH24h. This gene encodes a protein that belongs to the phosphatase $2 \mathrm{~A}$ regulatory subunit $\mathrm{B}$ family and is highly expressed in heart and skeletal muscle [43]. PPP2R5A is involved in the negative control of cell growth and division and plays a role in the Bio Systems Pathways that are directly involved in glycogen metabolism.

As suggested by Luo et al. [39], MYH3, a member of the $M Y H$ family, which contains the significant SNP ss478937995 on SSC12, is an interesting candidate gene for colour $\left(\mathrm{a}^{*}, \mathrm{~b}^{*}\right.$ and score), firmness and marbling. MYH is a large family of motor proteins that share various common features such as ATP hydrolysis, actin binding and potential for kinetic energy transduction. A missense mutation in $M Y H 3$ that induces an F4371 amino acid substitution, is known to segregate with distal arthrogryposis in a human family [44]. On SSC7, ELOVL2 at $233 \mathrm{~kb}$ downstream of the significant SNP ss478937524 can be regarded as another promising candidate gene for LM_Firmness, because it is involved in the metabolism of lipids and lipoproteins in mammals [45]. The KIF18A gene, which is positioned $71 \mathrm{~kb}$ away from the significant SNP ss131184920 on SSC2, may contribute to the LM_Firmness trait measured in DLY pigs. KIF18A is a member of the kinesin superfamily of microtubule-associated molecular motors that use the energy derived from ATP hydrolysis to produce force and movement along the microtubule $[46,47]$.

Based on the Erhualian dataset, four QTL that are involved in LM_MoistureContent were detected on SSC2, SSC8, SSC12 and SSC16. The significant SNP ss 131186989 on SSC2 is just $2.5 \mathrm{~kb}$ downstream from the site of the $L D H A$ gene, which encodes lactate dehydrogenase A that catalyzes the conversion of L-lactate and NAD to pyruvate and $\mathrm{NADH}$ in the final step of anaerobic glycolysis. LDHA is found predominantly in muscle tissue and belongs to the lactate dehydrogenase family [48]. On SSC16, the peak SNP ss131538960 was located between MFAP3 (microfibrillar-associated protein 3) and GALNT10 (polypeptide N-acetylgalactosaminyltransferase 10), within a region of $130 \mathrm{~kb}$. GALNT10 encodes a protein that may have increased catalytic activity towards glycosylated peptides compared to that towards nonglycosylated peptides [49], and, thus, it may be a better candidate gene than MFAP3. However, no obvious candidate genes were found for the other two loci on SSC8 and SSC12, where the nearest genes (ENSSSCG00000029791 and NFE2L1) have no clear functional annotations correlated with the phenotype.

The most significant SNP ss131398982 for LM ColorScore_36h in the DLY dataset is located only $47 \mathrm{~kb}$ away from the ASNS gene, which encodes a protein that is involved in the synthesis of asparagine. This gene complements a mutation in the temperature-sensitive hamster mutant ts11, which blocks progression through the G1 phase of the cell cycle at non-permissive temperatures [50]. Within the region between 127.41 and $134.39 \mathrm{Mb}$ on SSC15, the effects of the QTL on LM_ColorM_a36h and LM_ColorScore_36h observed for the DLY population may be partially or completely due to mutations in the PRKAG3 gene at $133.80 \mathrm{Mb}[51,52]$. To confirm this, further analyses combining data based on 60K SNPs and PRKAG3 sequence data are required.

Because precision of QTL location depends on various factors (e.g. marker density, population size, LD structure and precision of phenotypic measurement), it is necessary to be cautious when interpreting the results for loci with 95\% confidence intervals that are larger than $1 \mathrm{Mb}$. One cannot exclude that the genes that underlie the QTL are outside the $1 \mathrm{Mb}$ region that was investigated for candidate genes.

\section{Conclusions}

In conclusion, we performed GWAS analyses for meat quality traits in Chinese Erhualian and western DLY populations. Overall, 28 and nine SNPs surpassed the significance level in the Erhualian and DLY pig data, respectively. The strongest association was obtained between drip loss and a SNP on SSC4 for the Erhualian population. Comparison of the loci that were detected by GWAS of Erhualian and DLY pig data demonstrated that only a few QTL were common to both pig lines, which reflects the large differences in genetic architecture of meat quality traits between the Chinese and western breeds. Our results suggest that several QTL affect multiple traits among which some are reported for the first time. The meta-analysis that combined GWAS of four populations uncovered seven novel loci, which were not detected by single-population GWAS. These novel loci 
represent variants that may be shared between these populations. For each of the QTL regions detected, we identified several candidate genes based on the possible relationship between gene function and the corresponding trait. This study provides new insights into the genetic basis of meat quality traits in pigs and further investigations are needed to guide the application of these QTL in future swine breeding programs.

\section{Additional files}

Additional file 1: Figure S1. Quantile-quantile (Q-Q) plots of SNP distribution after quality control in GWAS for all tested meat quality traits. (A) Results for the Erhualian (EHL) population. (B) Results for the DLY population. Description: Datasets include 35985 and 56216 SNPs for the Erhualian and DLY populations, respectively. In the Q-Q plots, $-\log _{10} P$ values of observed association statistics on the $Y$-axis were compared to those of the association statistics expected under the hypothesis of no association on the X-axis. The solid line represents concordance between observed and expected values. The shaded region shows the 95\% confidence interval based on Beta distribution. Genomic inflation factor, $\lambda$, is shown for each dataset.

Additional file 2: Figure S2. Quantile-quantile (Q-Q) plots of SNP distribution after quality control in the GWAS meta-analysis. Description: The dataset includes 29516 SNPs that are common among the four pig populations: Erhualian, DLY, $F_{2}$ and Sutai. In the Q-Q plots, $-\log _{10} P$ values of observed association statistics on the $Y$-axis were compared to those of the association statistics expected under the hypothesis of no association on the X-axis. The solid line represents concordance between observed and expected values. The shaded region shows the $95 \%$ confidence interval based on Beta distribution. Genomic inflation factor, $\lambda$, is shown for each dataset.

Additional file 3: Figure S3. Manhattan plots of GWAS for meat quality traits on LM and SM from Chinese Erhualian pigs and on LM from Western DLY pigs. (A) Results for the Erhualian population. (B) Results for the DLY population. LM, longissimus muscle; SM: semimembranosus muscle. Description: In the plots, negative $\log _{10} P$ values of the quantified SNPs were plotted against their genomic positions. SNPs on different chromosomes are indicated by different colours. Dotted, dashed and solid lines correspond to the thresholds of: (A) 1.00E-04, 2.77E-05 and 1.38E-06, respectively; (B) 1.00E-04, 1.78E-05 and 8.89E-07, respectively.

Additional file 4: Figure S4. Manhattan plots of the GWAS meta-analysis for seven meat quality traits across four pig populations: Erhualian, DLY, White Duroc $\times$ Erhualian $F_{2}$ and Sutai. Description: In these plots, negative $\log _{10} P$ values of the quantified SNPs were plotted against their genomic positions. SNPs on different chromosomes are indicated by different colours. Dotted, dashed and solid lines correspond to the thresholds of 1.00E-04, 3.39E-05 and 1.69E-06, respectively.

\section{Competing interests}

The authors declare that they have no competing interest.

\section{Authors' contributions}

$X L$ performed the experiments, analyzed the data, prepared the figures and drafted the manuscript. XX performed the experiments and drafted the manuscript. JY and LZ performed the experiments. BY analyzed the data. HA established the Erhualian population. HM, XX, YH and SF performed the experiments. SX helped establish the experimental populations. JR performed the experiments. JM established the DLY and Sutai populations, performed the experiments, analyzed the data and revised the manuscript. $\mathrm{LH}$ conceived and designed the experiments. All authors read and approved the final manuscript

\section{Acknowledgments}

This research was supported by the Development Programs for Basic Research of China (973 Programs, No. 2012CB124702 and 2012CB722502), the Key Project of National Nature Science Foundation of China (No. 31230069) and the Cultivation Programs for Young Scientists of Jiangxi Province (No. 20133BCB23013).

Received: 16 October 2014 Accepted: 9 April 2015

Published online: 12 May 2015

\section{References}

1. Gallardo D, Pena RN, Quintanilla R, Ramirez O, Almuzara D, Noguera JL, et al. Quantitative trait loci analysis of a Duroc commercial population highlights differences in the genetic determination of meat quality traits at two different muscles. Anim Genet. 2012;43:800-4.

2. Moeller SJ, Miller RK, Edwards KK, Zerby HN, Logan KE, Aldredge TL, et al. Consumer perceptions of pork eating quality as affected by pork quality attributes and end-point cooked temperature. Meat Sci. 2010;84:14-22.

3. Nonneman DJ, Shackelford SD, King DA, Wheeler TL, Wiedmann RT, Snelling WM, et al. Genome-wide association of meat quality traits and tenderness in swine. J Anim Sci. 2013;91:4043-50.

4. Li HD, Lund MS, Christensen OF, Gregersen VR, Henckel P, Bendixen C. Quantitative trait loci analysis of swine meat quality traits. J Anim Sci. 2010;88:2904-12.

5. Rosenvold $\mathrm{K}$, Andersen HJ. Factors of significance for pork quality-a review. Meat Sci. 2003;64:219-37.

6. Andersson L, Georges M. Domestic-animal genomics: deciphering the genetics of complex traits. Nat Rev Genet. 2004;5:202-12.

7. Suzuki K, Irie M, Kadowaki H, Shibata T, Kumagai M, Nishida A. Genetic parameter estimates of meat quality traits in Duroc pigs selected for average daily gain, longissimus muscle area, backfat thickness, and intramuscular fat content. J Anim Sci. 2005;83:2058-65.

8. Van Laere AS, Nguyen M, Braunschweig M, Nezer C, Collette C, Moreau L, et al. A regulatory mutation in IGF2 causes a major QTL effect on muscle growth in the pig. Nature. 2003;425:832-6.

9. Oczkowicz M, Mucha A, Tyra M, Ropka-Molik K, Piorkowska K. Lack of the associations of the polymorphisms in IGF2, MC4R and GNAS genes with reproduction traits in pigs and imprinting analysis of IGF2 gene in ovary and cornus uteri. Reprod Domest Anim. 2013;48:562-8.

10. Uimari $P$, Sironen A. A combination of two variants in PRKAG3 is needed for a positive effect on meat quality in pigs. BMC Genet. 2014;15:29.

11. Fujii J, Otsu K, Zorzato F, De Leon S, Khanna VK, Weiler JE, et al. Identification of a mutation in porcine ryanodine receptor associated with malignant hyperthermia. Science. 1991;253:448-51.

12. Gao Y, Zhang R, Hu X, Li N. Application of genomic technologies to the improvement of meat quality of farm animals. Meat Sci. 2007;77:36-45.

13. Riquet J, Gilbert H, Servin B, Sanchez MP, lannuccelli N, Billon Y, et al. A locally congenic backcross design in pig: a new regional fine QTL mapping approach miming congenic strains used in mouse. BMC Genet. 2011;12:6.

14. Ramos AM, Crooijmans RP, Affara NA, Amaral AJ, Archibald AL, Beever JE, et al. Design of a high density SNP genotyping assay in the pig using SNPs identified and characterized by next generation sequencing technology. PLoS ONE. 2009:4, e6524.

15. Ren J, Mao H, Zhang Z, Xiao S, Ding N, Huang L. A 6-bp deletion in the TYRP1 gene causes the brown colouration phenotype in Chinese indigenous pigs. Heredity (Edinb). 2011;106:862-8.

16. Fan B, Onteru SK, Du ZQ, Garrick DJ, Stalder KJ, Rothschild MF. Genomewide association study identifies loci for body composition and structural soundness traits in pigs. PLoS ONE. 2011;6, e14726.

17. Munoz M, Rodriguez MC, Alves E, Folch JM, Ibanez-Escriche N, Silio L, et al. Genome-wide analysis of porcine backfat and intramuscular fat fatty acid composition using high-density genotyping and expression data. BMC Genomics. 2013;14:845.

18. Ma J, Ren J, Guo Y, Duan Y, Ding N, Zhou L, et al. Genome-wide identification of quantitative trait loci for carcass composition and meat quality in a large-scale White Duroc $x$ Chinese Erhualian resource population. Anim Genet. 2009:40:637-47.

19. Duan YY, Ma JW, Yuan F, Huang LB, Yang KX, Xie JP, et al. Genome-wide identification of quantitative trait loci for pork temperature, $\mathrm{pH}$ decline, and glycolytic potential in a large-scale White Duroc x Chinese Erhualian resource population. J Anim Sci. 2009:87:9-16.

20. Duan Y, Zhou L, Ma J, Guo B, Huang W, et al. Genome-wide identification of quantitative trait loci for pork firmness in a large-scale White Duroc $\times$ Chinese Erhualian resource population. Chinese J Anim Vet Sci. 2009;40:6-11. 
21. Ren J, Guo YM, Ma JW, Huang LS. Growth and meat quality QTL in pigs with special reference to a very large Erhualian $\times$ White Duroc resource population. In: Proceedings of 8th World Congress on Genetics Applied to Livestock Production: 13-18 August 2006; Belo Horizonte. 2006.

22. Zhou L, Guo Y, Duan Y, Zhang Z, Yang K, et al. QTL mapping for drip loss in a White Duroc $\times$ Erhualian resource population. Agric Sci China. 2011;44:2131-8.

23. Yang B, Zhang W, Zhang Z, Fan Y, Xie X, Ai H, et al. Genome-wide association analyses for fatty acid composition in porcine muscle and abdominal fat tissues. PLoS ONE. 2013;8, e65554.

24. Zhang Z, Hong Y, Gao J, Xiao S, Ma J, Zhang W, et al. Genome-wide association study reveals constant and specific loci for hematological traits at three time stages in a White Duroc x Erhualian F2 resource population. PLoS ONE. 2013;8, e63665.

25. Bao WB, Ye L, Pan ZY, Zhu J, Zhu GQ, Huang XG, et al. Beneficial genotype of swine FUT1 gene governing resistance to E. coli F18 is associated with important economic traits. J Genet. 2011;90:315-8.

26. Ma J, Yang J, Zhou L, Zhang Z, Ma H, Xie X, et al. Genome-wide association study of meat quality traits in a white DurocxErhualian F2 intercross and Chinese Sutai pigs. PLoS ONE. 2013;8, e64047.

27. Rassmussen AJ, Andersson M. New method for determination of drip loss in pork muscles. In: Proceedings of the 42nd International Congress of Meat Science and Technology:1-6 September 1996; Lillehammer. 1996. p. 286-7.

28. Huff-Lonergan E, Lonergan SM. New frontiers in understanding drip loss in pork: recent insights on the role of postmortem muscle biochemistry. J Anim Breed Genet. 2007;124:19-26.

29. Otto G, Roehe R, Looft H, Thoelking L, Kalm E. Comparison of different methods for determination of drip loss and their relationships to meat quality and carcass characteristics in pigs. Meat Sci. 2004;68:401-9.

30. Berg EP. Pork composition and quality assessment procedures. 1st ed. Des Moiners: National Pork Producer Council (NPPC); 2006.

31. Aulchenko YS, Ripke S, Isaacs A, Van Duijn CM. GenABEL: an R library for genome-wide association analysis. Bioinformatics. 2007;23:1294-6.

32. Breslow NE, Clayton DG. Approximate inference in generalized linear mixed models. J Am Stat Assoc. 1993;88:9-25.

33. Yu J, Pressoir G, Briggs WH, Vroh Bi I, Yamasaki M, Doebley JF, et al. A unified mixed-model method for association mapping that accounts for multiple levels of relatedness. Nat Genet. 2005;38:203-8.

34. Hayes BJ, Goddard ME. Technical note: prediction of breeding values using marker-derived relationship matrices. J Anim Sci. 2008;86:2089-92.

35. Lander E, Kruglyak L. Genetic dissection of complex traits: guidelines for interpreting and reporting linkage results. Nat Genet. 1995;11:241-7.

36. Yang Q, Cui J, Chazaro I, Cupples LA, Demissie S. Power and type I error rate of false discovery rate approaches in genome-wide association studies. BMC Genet. 2005;6:S134.

37. Pearson TA, Manolio TA. How to interpret a genome-wide association study. JAMA. 2008;299:1335-44

38. Willer CJ, Li Y, Abecasis GR. METAL: fast and efficient meta-analysis of genomewide association scans. Bioinformatics. 2010;26:2190-1.

39. Luo W, Cheng D, Chen S, Wang L, Li Y, Ma X, et al. Genome-wide association analysis of meat quality traits in a porcine Large White $x$ Minzhu intercross population. Int J Biol Sci. 2012;8:580-95.

40. Amaral AJ, Megens HJ, Crooijmans RP, Heuven HC, Groenen MA. Linkage disequilibrium decay and haplotype block structure in the pig. Genetics. 2008;179:569-79.

41. Ai H, Huang L, Ren J. Genetic diversity, linkage disequilibrium and selection signatures in chinese and Western pigs revealed by genome-wide SNP markers. PLoS ONE. 2013:8, e56001.

42. loannidis JP, Thomas G, Daly MJ. Validating, augmenting and refining genome-wide association signals. Nat Rev Genet. 2009;10:318-29.

43. McCright B, Virshup DM. Identification of a new family of protein phosphatase 2A regulatory subunits. J Biol Chem. 1995;270:26123-8.

44. Alvarado DM, Buchan JG, Gurnett CA, Dobbs MB. Exome sequencing identifies an MYH3 mutation in a family with distal arthrogryposis type 1. J Bone Joint Surg Am. 2011;93:1045-50.

45. Jakobsson A, Westerberg R, Jacobsson A. Fatty acid elongases in mammals: their regulation and roles in metabolism. Prog Lipid Res. 2006;45:237-49.

46. Stumpff J, Wagenbach M, Franck A, Asbury CL, Wordeman L. Kif18A and chromokinesins confine centromere movements via microtubule growth suppression and spatial control of kinetochore tension. Dev Cell. 2012;22:1017-29.
47. Mayr MI, Hummer S, Bormann J, Grüner T, Adio S, Woehlke G, et al. The human kinesin Kif18A is a motile microtubule depolymerase essential for chromosome congression. Curr Biol. 2007;17:488-98.

48. Maekawa M, Sudo K, Kanno T, Li SS. Molecular characterization of genetic mutation in human lactate dehydrogenase-A (M) deficiency. Biochem Biophys Res Commun. 1990;168:677-82.

49. Perrine CL, Ganguli A, Wu P, Bertozzi CR, Fritz TA, Raman J, et al. Glycopeptide-preferring polypeptide GalNAc transferase 10 (ppGalNAc T10), involved in mucin-type O-glycosylation, has a unique GalNAc-O-Ser/Thr-binding site in its catalytic domain not found in ppGalNAc T1 or T2. J Biol Chem. 2009;284:20387-97.

50. Iwamoto S, Mihara K, Downing JR, Pui CH, Campana D. Mesenchymal cells regulate the response of acute lymphoblastic leukemia cells to asparaginase. J Clin Invest. 2007;117:1049-57.

51. Milan D, Jeon JT, Looft C, Amarger V, Robic A, Thelander M, et al. A mutation in PRKAG3 associated with excess glycogen content in pig skeletal muscle. Science. 2000;288:1248-51.

52. Ciobanu D, Bastiaansen J, Malek M, Helm J, Woollard J, Plastow G, et al. Evidence for new alleles in the protein kinase adenosine monophosphate-activated gamma(3)-subunit gene associated with low glycogen content in pig skeletal muscle and improved meat quality. Genetics. 2001;159:1151-62.

\section{Submit your next manuscript to BioMed Central and take full advantage of:}

- Convenient online submission

- Thorough peer review

- No space constraints or color figure charges

- Immediate publication on acceptance

- Inclusion in PubMed, CAS, Scopus and Google Scholar

- Research which is freely available for redistribution 